\title{
Entorhinal cortex lesions as a model of age-related changes in hippocampal function
}

\author{
HEATHER N. FUGGER, JULIE M. LICHTENVOORT, and THOMAS C. FOSTER \\ University of Virginia, Charlottesville, Virginia
}

\begin{abstract}
This study investigated unilateral entorhinal cortex lesions as a model of age-related changes in hippocampal function relative to more extensive bilateral lesions previously employed. Spatial memory and hippocampal physiology were examined in male rats ( 3 months) following bilateral or unilateral entorhinal cortex lesions, or in sham controls. All groups exhibited cue discrimination in a water escape task. Only control and unilaterally lesioned rats exhibited acquisition of the spatial version of the task. A probe trial administered $24 \mathrm{~h}$ after spatial training demonstrated impaired retention for unilaterally lesioned animals. Lesions decreased perforant path fiber potentials and synaptic responses; however, compensatory modifications of surviving perforant path afferents were observed for unilaterally lesioned animals. Behavioral and physiological changes following unilateral lesions were similar to those observed during aging, indicating that partial lesions provide a better model of aging than do bilateral lesions. In addition, the results emphasize retention as a cognitive constituent of the hippocampalentorhinal system.
\end{abstract}

Age-related impairments in cognitive functions have been well documented for several species, including humans. A common finding is that aged rats learn and retain information over short intervals, but exhibit impaired retention when tested over longer intervals (e.g., Foster, Barnes, Rao, \& McNaughton, 1991; Gold, McGaugh, Hankins, Rose, \& Vasquez, 1981; Mabry, McCarty, Gold, \& Foster, 1996; Martinez \& Rigter, 1980; Solomon \& Pendlebury, 1994; Winocur, 1988; Zornetzer, Thompson, \& Rogers, 1982). Of particular vulnerability in the aged animal is memory performance on tasks that are thought to depend on an intact hippocampus, including deficits in the retention of information across a delay period and processing of spatial information (Geinisman, deToledoMorrell, Morrell, \& Heller, 1995). For example, on the spatial version of the Morris water task, aged rats have been shown to exhibit similar levels of acquisition as young adults either with massed training or following extensive distributed training. However, these rats have been shown to display a more rapid rate of forgetting for spatial discrimination (Foster et al., 1991; Mabry et al., 1996; Rapp, Rosenberg, \& Gallagher, 1987). One possible cause of impaired memory is a loss of hippocampal neurons; however, recent work indicates no difference in hippocampal cell number between aged rats that are memory impaired and memory unimpaired on this task (Rasmussen, Schliemann, Sørensen, Zimmer, \& West, 1996). Conversely, evidence for loss of hippocampal input from the perforant path of aged memory-impaired rats has been provided in

The authors wish to thank Nick Waters for advice on statistical analyses, and Chris Norris and Ted Dumas for comments on the manuscript. Correspondence should be addressed to T. C. Foster, Department of Psychology, University of Virginia, Charlottesville, VA 22903 (e-mail: tcf5a@virginia.edu). both anatomical (Geinisman et al., 1995) and physiological (Barnes \& McNaughton, 1980; Foster et al., 1991) realms. These results suggest that age-related memory impairments may be due to loss of hippocampal afferents.

Specific lesions within the hippocampal system (hippocampus, subiculum, entorhinal cortex, or medial septum) have been used to study the nature of age-related memory impairments (Olton \& Wenk, 1987). The underlying assumption is that destruction of a specific array of neurons or afferents will have functional repercussions analogous to normal aging. Descriptions of behavioral impairments following lesions can then be used to support hypotheses that specific patterns of atrophy/irregularity in the brain contribute to memory deficits. The advantage of a lesion model of aging is that the time course of physiological changes following deafferentation can be specified. Therefore, the model permits characterization of compensatory mechanisms due to afferent loss, which may be ongoing in aged animals, and provides a means for assessing intervention treatments. However, significant quantitative and qualitative differences may distinguish age-related impairments from lesion-induced deficits. Complete removal of either the hippocampus or the hippocampal afferents results in profound deficits in the acquisition of spatial information (Olton \& Wenk, 1987). In contrast to aging studies, which indicate subtle differences in acquisition and poor retention over extended durations, studies of bilateral lesions of the hippocampus or the entorhinal cortex have shown that such lesions impair learning of the spatial discrimination version of the Morris water task (Nagahara, Otto, \& Gallagher, 1995; Schenk \& Morris, 1985; Skelton \& McNamara, 1992).

Behavioral differences during aging and following lesion-induced hippocampal deafferentation may relate to the extent of cell loss under the two conditions and the 
capacity of surviving inputs to reorganize and modify synaptic contacts. Damage to perforant path afferents has been employed extensively as a model of lesioninduced synaptic plasticity in the hippocampus. Following unilateral destruction of the entorhinal cortex or perforant path, surviving afferents extend to the vacated portions of the granule cell dendrites in the outer molecular layer (Geddes et al., 1985; Lynch, Gall, Rose, \& Cotman, 1976; Scheff, Bernardo, \& Cotman, 1980; Steward, Cotman, \& Lynch, 1976). Transmission efficacy may increase following collateral sprouting and reinnervation by surviving perforant path fibers. The reinnervation and increased synaptic strength may account for the behavioral recovery over time that has been observed following partial or unilateral lesions (Loesche \& Steward, 1977; Reeves \& Smith, 1987). These results suggest that afferent loss, such as that observed in aged animals or following unilateral lesions, may be compensated for by remodeling of the strength or connectivity of the surviving brain circuitry. Thus, a unilateral lesion of the entorhinal cortex may provide a better model of the behavioral and physiological adjustments of aged animals relative to previous bilateral lesion models.

In this context, Hagan, Verheijck, Spigt, and Ruigt (1992) reported that rats with bilateral ibotenic acid lesions of the entorhinal cortex exhibited spatial learning on the water escape task. However, these rats also demonstrated impaired escape latencies for longer delays (several hours), suggesting increased forgetting of spatial information. Ibotenic acid may have produced incomplete lesions in such a way that remaining cells were sufficient to enable animals to improve over the course of training within a day (Nagahara et al., 1995). Similarly, unimpaired learning and rapid forgetting have been reported during normal aging in humans and rats (Foster et al., 1991; Huppert \& Kopelman, 1989; Mabry et al., 1996). In aged humans and rats, loss of perforant path input may be compensated for by synaptic plasticity mechanisms at surviving synapses through a postsynaptic increase in transmitter responsiveness or neuronal sprouting (Barnes, Rao, Foster, \& McNaughton, 1992; Bertoni-Freddari, Fattoretti, Pieroni, \& Meier-Ruge, 1992; Foster et al., 1991; Hyman, Kromer, \& Van Hoesen, 1987). Together, these results support consideration of a partial lesion, over bilateral lesions, as a model of the behavioral and physiological modifications of aged memory-impaired animals.

\section{METHOD}

\section{Subjects}

Three-month-old male Fischer-344 rats $(250-350 \mathrm{~g})$ were individually housed in opaque plastic cages with food and water available ad libitum and maintained on a 12:12-h light:dark cycle. All surgeries and behavioral testing occurred during the light phase of the cycle. Animals were handled for at least 2 weeks prior to surgical procedures.

\section{Surgery}

Rats were anesthetized with sodium pentobarbital (Nembutal; $50 \mathrm{mg} / \mathrm{kg}$ ). An electrode was positioned stereotaxically to create unilateral (UNI; $n=8$ ) or bilateral (BIL; $n=5$ ) lesions of the en- torhinal cortex $\left(10^{\circ}\right.$ angle, $4.1 \mathrm{~mm}$ from lambda, at depths of 2.0 , 4.0 , and $6.0 \mathrm{~mm}$ ventral to the surface of the dura). In the case of UNI lesions, the side that sustained the lesion was randomized across animals. Electrolytic lesions were induced by a $1-\mathrm{mA}$ anodal current for $45 \mathrm{sec}$ at each depth. In the case of sham-operated controls (CON; $n=7$ ), electrodes were lowered in the absence of current application. Animals were allowed to recover for 3 weeks before behavioral testing.

\section{Behavior}

Animals were tested on both spatial and nonspatial versions of the Morris swim task (Foster et al., 1991). Animals were tested in a black tank, $1.7 \mathrm{~m}$ in diameter, positioned in a well-lighted room containing (when appropriate) an assortment of 2- and 3-D cues (e.g., ladder, posters, lamp). Water was maintained at a temperature of $26^{\circ} \pm 2^{\circ} \mathrm{C}$, at a level approximately $8 \mathrm{~cm}$ below the edge of the tank. Behavioral data were acquired with a Columbus Instruments tracking system and included escape latency for all trials and differential quadrant search time for probe trials in which the escape platform was removed.

Three weeks post surgery, the cue discrimination task was administered. In this phase, the black platform was made visible by affixing a white band around the top to increase contrast between it and the black pool. The platform was placed in the center of one quadrant in the tank and allowed to extend $1.5 \mathrm{~cm}$ above the water level. No intentional cues other than the platform were available to the subject during this phase of training. Black curtains were hung around the pool to limit distal cues. Immediately prior to the first cue training trial, animals were habituated to the pool by allowing them a $30-\mathrm{sec}$ free swim, $30 \mathrm{sec}$ on the escape platform $(17 \mathrm{~cm}$ in diameter), and practice climbing onto the platform from the pool. Following habituation training, rats received six blocks of three trials (maximum $60 \mathrm{sec}$ each) with an intertrial interval (ITI) of $30 \mathrm{sec}$ and an interblock interval of $15 \mathrm{~min}$. On each trial, rats were placed in the pool facing the wall from one of eight equally spaced start locations. Platform and start locations were randomized across each trial. If the rat found the escape platform within the $60 \mathrm{sec}$, it remained on the platform for the 30 -sec ITI. If the rat failed to find the platform in the allotted time, it was gently placed on the platform for the ITI.

Three days following the cue discrimination training, the spatial discrimination task was administered. A black escape platform was located approximately $1.5 \mathrm{~cm}$ beneath the water level and remained in the same location relative to distal cues in the room throughout testing. Place training, massed into 1 day, consisted of six blocks of three trials, with an ITI of $30 \mathrm{sec}$ and an interblock interval of $15 \mathrm{~min}$. Rats were initially placed on the platform for $30 \mathrm{sec}$ prior to the first trial. For each trial, the rat was placed in the water facing the wall of the tank and allowed to swim for a maximum of $60 \mathrm{sec}$. After locating the platform, the rat remained on the platform during the extent of the ITI. If the rat failed to find the platform in the allotted time, it was placed onto the platform by the experimenter for the ITI.

Probe trials were employed to determine whether groups could acquire and retain spatial discrimination in performance of the water escape task. The probe trial consisted of placing the animal in the pool, without the platform, for $1 \mathrm{~min}$ and recording the time the animal spent searching each quadrant. One probe trial was administered 15 min after Block 6 of the spatial discrimination task. Following completion of this probe trial, a refresher block of three training trials was administered (i.e., Block 7). A second probe trial to determine retention was delivered $24 \mathrm{~h}$ after the last training trial.

\section{Electrophysiology}

Two to seven weeks after behavioral testing, animals were sacrificed for in vitro electrophysiological recordings. Electrophysiological recordings were carried out at the same time for all animals to minimize any variance attributable to circadian rhythms. Animals 
were deeply anesthetized with methoxyflurane and decapitated. The brains were quickly removed and placed in oxygenated $(95 \%$ $\mathrm{O}_{2}, 5 \% \mathrm{CO}_{2}$ ) artificial cerebrospinal fluid (ACSF) containing (in $\mathrm{mM}$ ) the following: $\mathrm{NaCl} 124, \mathrm{KCl} 2, \mathrm{KH}_{2} \mathrm{PO}_{4} 1.25, \mathrm{MgSO}_{4} 2$, $\mathrm{CaCl}_{2} 2, \mathrm{NaHCO}_{3} 26$, and dextrose 10 , with pH 7.4. During brain removal and hippocampal dissection, the brains were visually inspected for signs of lesion damage. In all cases, damage included the medial aspects of the entorhinal cortex, with variable damage extending to the lateral portions of the entorhinal cortex. No evidence of midbrain or perirhinal damage was observed. Hippocampi were dissected and slices $(400-500 \mu \mathrm{m})$ from the most dorsal half of the hippocampus (i.e., the septal pole) were cut parallel to the alvear fibers, using a gravity controlled tissue chopper. Slices were transferred to a standard interface recording chamber and continuously perfused $(1 \mathrm{ml} / \mathrm{min})$ at $30^{\circ} \mathrm{C}$ with oxygenated ACSF. Slices were permitted to equilibrate for at least $1 \mathrm{~h}$ before stimulation and recording were initiated.

A recording electrode constructed from a glass micropipette (1-6 M $\Omega$ ), pulled from thin-wall tubing $(1 \times .75 \mathrm{~mm})$ and filled with ACSF, was positioned in the middle third of the outer molecular layer of the dentate gyrus in order to record medial perforant path input to the granule cells. The stimulating electrode, consisting of a pair of insulated platinum-iridium wires, was positioned $1 \mathrm{~mm}$ away on either side of the dendritic recording electrode in order to activate medial perforant path fibers. Stimulation consisted of paired pulses (50-msec interstimulus interval) of $100-\mu \mathrm{sec}$ biphasic, constant-current stimulation. Paired pulses were delivered once every $30 \mathrm{sec}$. An input output (I/O) curve of the perforant path synaptic strength was constructed using five intensities $(100,150$, 200,250 , and $300 \mu \mathrm{A}$ ). Field potentials were amplified 100 times, band-pass filtered between $1 \mathrm{~Hz}$ and $1 \mathrm{kHz}$ by a differential $\mathrm{AC}$ amplifier (A-M Systems, Everett, WA), converted to digital units, and stored on a computer for off-line analysis.

Fiber potential amplitude and excitatory postsynaptic potential (EPSP) slope were extracted from the field potential records across the $\mathrm{I} / \mathrm{O}$ curve. The fiber potential amplitude was measured as the difference between two cursors set just prior to fiber potential onset and at the peak of the negative-going fiber potential. The slope of the field EPSP ( $\mu \mathrm{V} / \mathrm{msec})$ was determined as the difference between two cursors, separated by $1 \mathrm{msec}$, and placed on the middle portion of the descending phase of the EPSP. Data from a minimum of three slices per animals were collected and averaged, and the means were used as data points for subsequent analysis.

\begin{abstract}
Analyses
For acquisition on the cue and spatial tasks, latency measures were compared across blocks of trials using a repeated measures analysis of variance (ANOVA). Similarly, a repeated measures ANOVA on the EPSP slope measures was employed to examine the main effects of treatment and stimulus intensity across the $\mathrm{I} / \mathrm{O}$ curves. Post hoc Scheffé tests $(p<.05)$ were employed to locate specific differences. To ascertain whether the rats engaged in spatially selective search behavior during probe trials, Student $t$ tests were employed to determine whether the percent time searching the goal quadrant and quadrant opposite the goal were different from chance (i.e., $25 \%$ ).
\end{abstract}

\section{RESULTS}

\section{Behavior}

Figure 1 illustrates the mean latencies to escape on the cue version of the water task. A repeated measures ANOVA indicated a significant decrease in latency across trial blocks $[F(5,85)=31.224, p<.001]$ and no group effect or block $\times$ treatment interaction. The results indi-

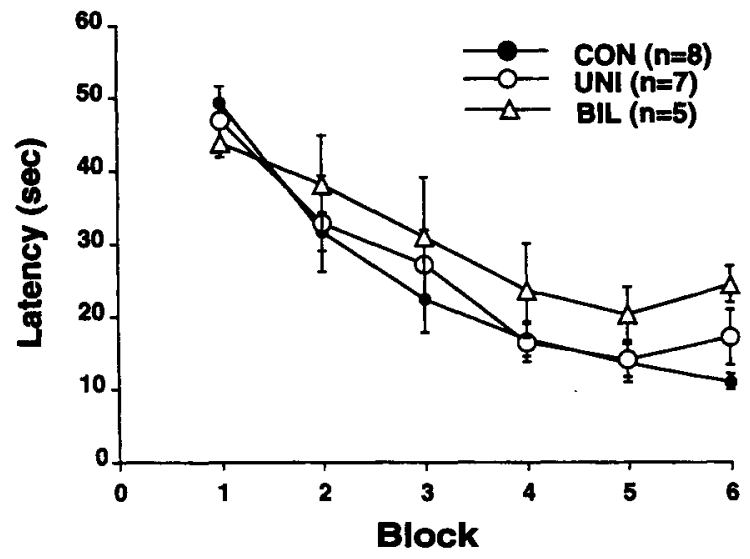

Figure 1. Entorhinal cortex lesions do not impair acquisition of cue discrimination. Mean latency to find the platform on the cued version of the water escape task for the control (CON), the unilateral (UNI), and the bilateral (BIL) groups. Each block consisted of three trials. Bars equal $S E M$.

cated that all groups exhibited a similar improvement in performance over the course of training.

Latency to find the platform on the spatial discrimination task for the three treatment groups is shown in Figure 2. Overall, latency measures were highest for the BIL group, followed by the UNI and CON animals. A repeated measures ANOVA on escape latency indicated a significant effect of group $[F(2,102)=9.378, p<.01]$ and trial block $[F(6,102)=3.876, p<.01]$, and no interaction. Post hoc comparisons indicated a significantly greater escape latency for BIL relative to CON for Blocks 3-6. The escape latencies for animals from the UNI group were not different from those from the BIL group and were increased relative to those of the CON group only on Block 3.

To test whether animals were employing a spatial discrimination strategy, a 60 -sec probe trial was presented approximately $15 \mathrm{~min}$ following completion of Block 6 . Comparisons of percent search time versus chance (i.e., $25 \%$ ) were conducted for the goal and opposite quadrants. As illustrated in Figure $3 \mathrm{~A}$, relative to chance, $\mathrm{CON}$ and UNI animals spent significantly more time searching the quadrant that had previously contained the escape platform [CON, $t(7)=5.82, p<.001 ; \mathrm{UNI}, t(6)=4.81, p<$ $.005]$ and spent significantly less time searching the opposite quadrant [CON, $t(7)=6.27, p<.005$; $\mathrm{UNI}, t(6)=$ $4.29, p<.01]$. In contrast, the search patterns for BIL animals were not different from chance for the two quadrants. The results indicate that CON and UNI rats learned to discriminate the spatial location of the escape platform.

Twenty-four hours following administration of the immediate probe trial, a second probe trial was given in order to measure retention. In contrast to the results of the immediate probe trial, Student $t$ tests of percent search time, relative to chance, indicated that only $\mathrm{CON}$ animals spent significantly more time in the goal quadrant $[t(7)=2.70$, 


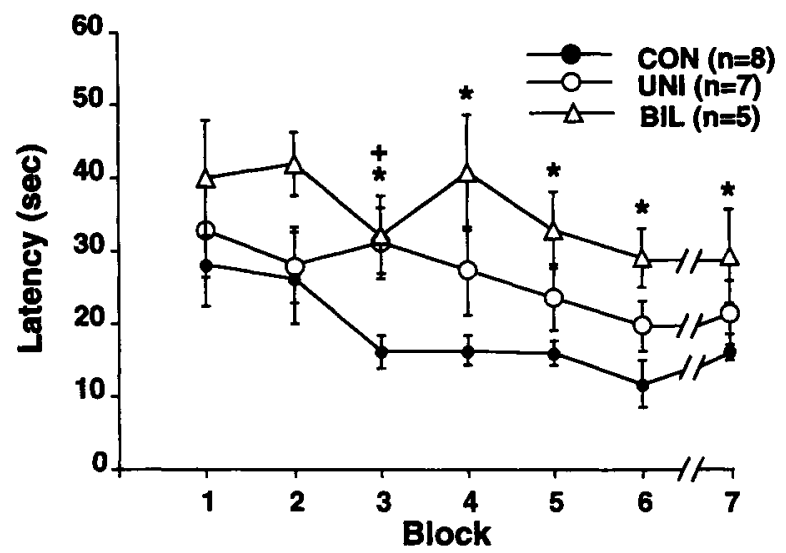

Figure 2. Mean latency on the spatial version of the water escape task for the control (CON), the unilateral (UNI), and the bilateral (BIL) groups. As in the cued version, each block consisted of three trials and training occurred all in 1 day. The break in the $x$-axis between Blocks 6 and 7 indicates the time point at which a probe trial was administered to measure acquisition (see Figure 3). Asterisks and plus sign indicate a significant difference between BIL and CON, and UNI and CON, respectively. Bars equal $S E M$.

$p<.05]$ and less time searching the opposite quadrant $[t(7)=4.23, p<.005]$ (Figure 3B). The results indicate that only CON animals continued to exhibit a marked spatial search strategy, suggesting that these animals retained the discrimination across the 24-h period.

\section{Electrophysiology}

Illustrated in Figure 4 are the I/O curves of synaptic responses elicited by perforant path stimulation and recorded in the outer molecular layer of the dentate gyrus. These recordings were performed ipsilateral to the lesion for UNI, and bilaterally for CON and BIL animals. An ANOVA of the mean EPSP slope across the five stimulus intensities revealed a significant effect of lesion $[F(2,68)=6.79, p<.01]$, stimulation intensity $[F(4,68)=45.76, p<.005]$, and an interaction between group and stimulation intensity $[F(8,68)=3.53, p<$ $.005]$. Post hoc tests of simple effects indicated that EPSP slope for BIL was significantly reduced in comparison to that for CON at all levels of the I/O curve. EPSP slope for $\mathrm{UNI}$ was significantly less than CON at 250 and $300 \mu \mathrm{A}$. The responses of BIL animals were not different from those of UNI animals at any level of the I/O curve.

In many instances, accurate measurement of the fiber potential could not be obtained for animals with electrolytic lesions, particularly at lower stimulation intensities. Therefore, fiber potential amplitude and measures of synaptic strength (EPSP slope/fiber potential amplitude) were examined only at the highest stimulation intensity. An ANOVA of fiber potentials elicited by $300-\mu \mathrm{A}$ stimulation revealed a significant effect of treatment $[F(2,19)$ $=18.80, p<.0005]$ (Figure 5A). Scheffé post hoc comparisons indicated that the fiber potential was reduced for both lesion groups relative to the $\mathrm{CON}$ group. In gen- eral, the EPSP/fiber potential ratio was larger for the UNI group than for the CON and BIL groups; however, an ANOVA indicated only a nonsignificant tendency for increased synaptic strength of the UNI animals $[F(2,19)=$ $2.92, p=.08]$ (Figure 5B).

The smaller synaptic responses of lesioned animals, evoked at the lower stimulation intensities, can render measures of paired-pulse facilitation unreliable (Dumas $\&$ Foster, 1995). Furthermore, the paired-pulse ratio for perforant path synapses may be a function of the EPSP amplitude (Moser, 1996; however, see Foster, Gagne, \& Massicotte, 1996). In order to compare paired-pulse ratios using similar amplitude EPSPs, the EPSP slopes of $\mathrm{CON}$ animals that approximated the mean evoked by $300-\mu \mathrm{A}$ stimulation for lesioned animal groups (EPSP
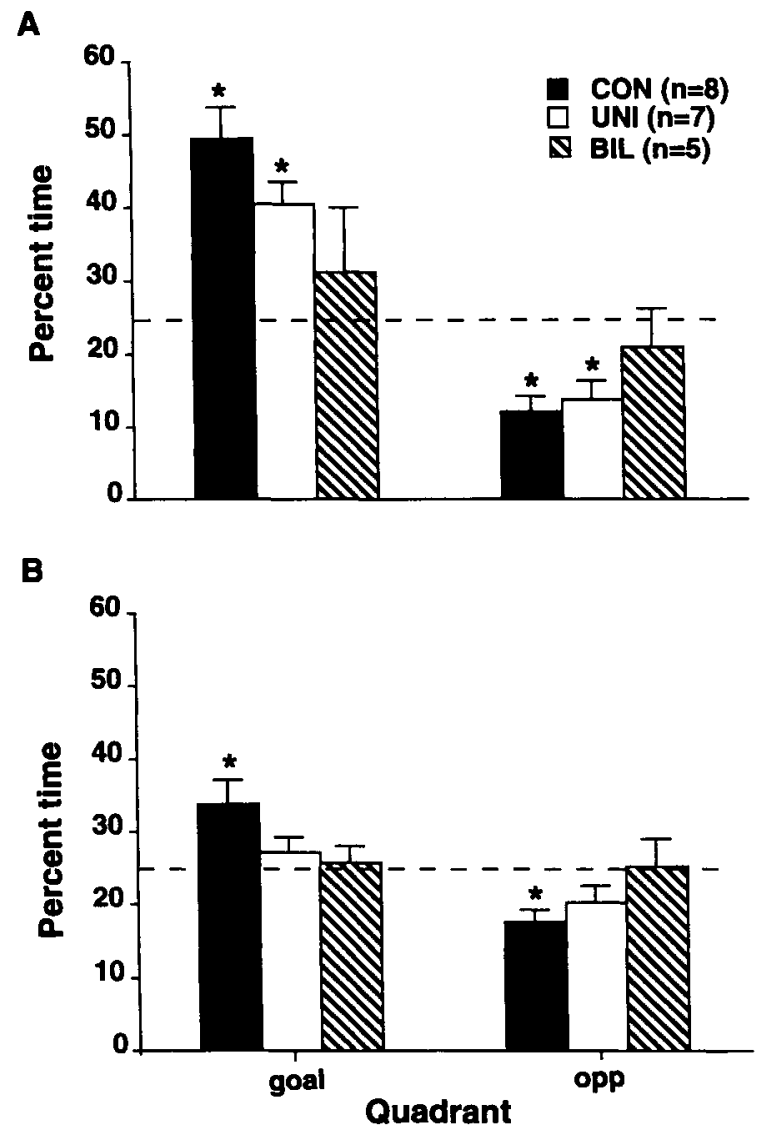

Figure 3. Searching behavior during testing of acquisition and retention of spatial discrimination for the control (CON), the unilateral (UNI), and the bilateral (BIL) groups. (A) Following Block 6 of the spatial version of the water task, a probe trial was administered to measure acquisition. The mean percent time spent searching the goal quadrant (goal) and the quadrant opposite the goal (opp) is illustrated for each group. CON and UNI groups spent significantly more time in the goal quadrant and significantly less time in the quadrant opposite the goal. (B) Twentyfour hours following the first probe trial, a second probe trial was administered to test for retention. Only control animals spent significantly more time in the goal quadrant and significantly less time in the quadrant opposite the goal. Asterisks indicate significant difference from chance (dashed line). Error bars equal $S E M$. 


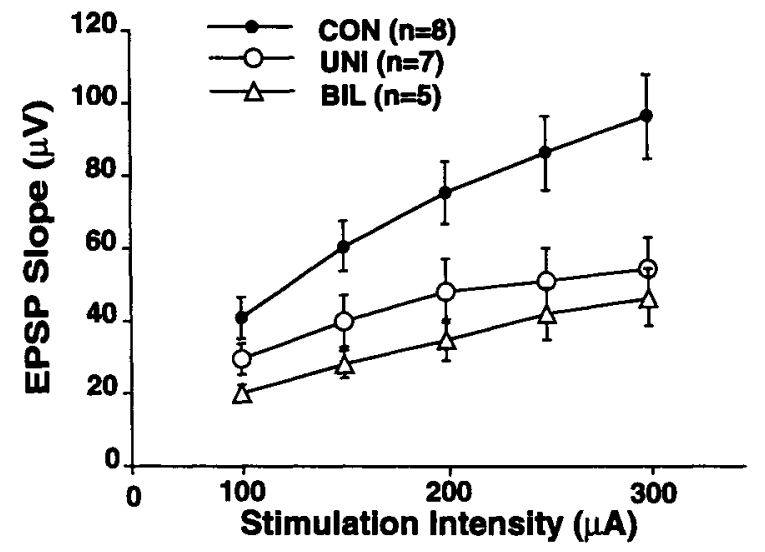

Figure 4. Input-output relationship for synaptic responses elicited by perforant path stimulation and recorded in the outer molecular layer of the dentate gyrus. Each point represents the mean excitatory postsynaptic potential (EPSP) slope for the control (CON), the unilateral (UNI), and the bilateral (BIL) groups at the given stimulus intensity. Bars equal $S E M$.

slope: $\mathrm{CON}=520.2 \pm 39.7 ; \mathrm{UNI}=543.4 \pm 84.3 ; \mathrm{BIL}=$ $460.9 \pm 78.8$ ) were employed. For CON animals, the EPSP slopes used in this comparison had been elicited at a stimulation intensity of 100-150 $\mu \mathrm{A}$. Separate ANOVAs indicated no difference in the EPSP slope for the conditioning pulse; however, a significant difference for the paired-pulse ratios was observed $[F(2,19)=4.05, p<$ $.05]$ (paired-pulse ratio mean $\pm S E M$ : CON, .86 \pm .06 ; UNI, $1.04 \pm .04$; BIL, $.99 \pm .02$ ). Scheffé post hoc comparisons indicated a significant difference between CON and UNI groups.

\section{DISCUSSION}

The present study indicates that bilateral removal of perforant path afferents disrupts acquisition of spatial information as determined by the Morris water escape task. This conclusion is similar to that previously reported by Schenk and Morris (1985) for electrolytic lesions and by Nagahara et al. (1995) following bilateral entorhinal cortex aspiration lesions and suggests that the entorhinal cortex is necessary for normal acquisition of spatial information when variable start positions are employed. The results seem to contradict the findings of Hagan et al. (1992), who failed to observe a learning deficit in rats following bilateral excitotoxic lesions directed at the entorhinal cortex. Differences between the performance of bilaterally lesioned animals in the present study and that in the Hagan et al. study may relate to the extent of hippocampal afferents remaining and the differences in the behavioral protocol. In the present study, with short ITIs, rats with unilateral lesions exhibited relatively normal acquisition performance during training and normal spatial search strategies on the probe trial, examined immediately after training. However, these animals displayed impaired retention of spatial information on a probe trial $24 \mathrm{~h}$ after the initial training. For the Hagan et al. study, brain-damaged animals also exhibited selective spatial search patterns when tested immediately following training; however, there was a tendency toward group differences on the first trial of each training block during the initial spatial training, and performance deficits of lesioned animals became evident on the first trial for training blocks during relearning of the spatial task. As noted by the authors, the deficits may relate to the delay of several hours between training blocks, suggesting impaired retention.

Age-related deficits in the performance of spatial discrimination are also sensitive to the distribution of trials, with age-related impairments becoming pronounced as the interval between trials is increased (Diana, Domenici, Scotti de Carolis, Loizzo, \& Sagratella, 1995; Foster et al., 1991; Gage, Dunnett, \& Bjorklund, 1984; Linder, Balch, \& VanderMaelen, 1992; Linder \& Gribkoff, 1991; Mabry et al., 1996; Rapp et al., 1987; Rasmussen et al., 1996). In fact, when training sessions are administered across a number of days, a characteristic "saw-toothed" pattern of behavior emerges for aged rats during the first

A

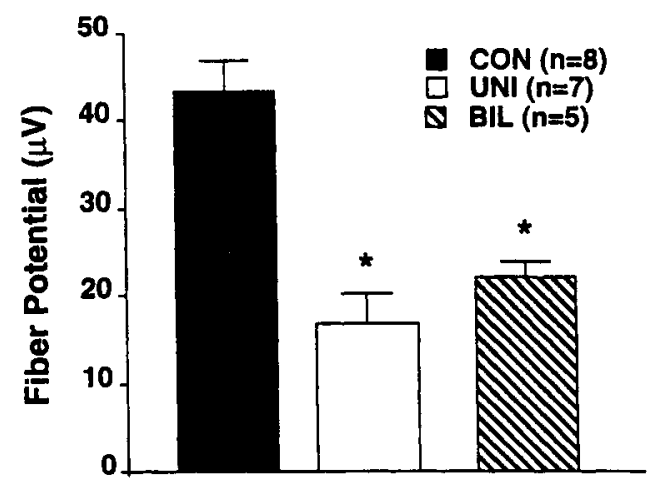

B

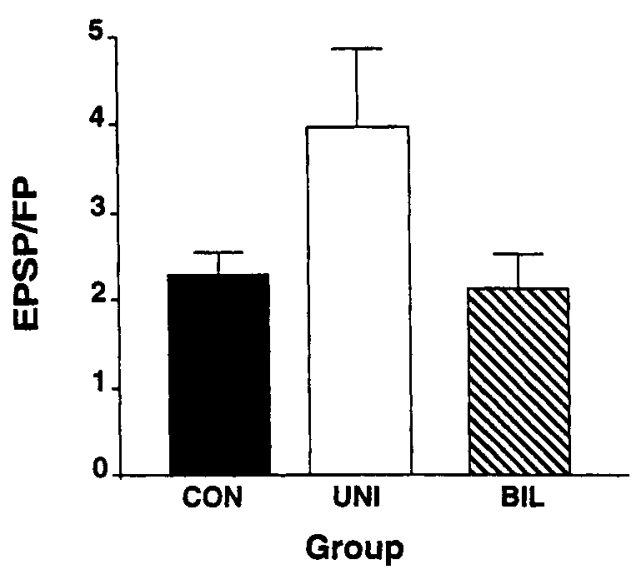

Figure 5. (A) Mean fiber potential amplitude at the highest stimulation intensity $(300 \mu \mathrm{A})$. Fiber potentials for the unilateral (UNI) and the bilateral (BIL) groups were significantly reduced compared with those for the control (CON) group. (B) Mean synaptic strength for a given excitatory postsynaptic potential (EPSP) slope $(\sim 500 \mu \mathrm{V})$. Synaptic strength was measured as the ratio of EPSP slope/fiber potential amplitude. Bars equal $S E M$. 
several days of training (Diana et al., 1995; Gage et al., 1984; Rapp et al., 1987). This pattern appears as an improved performance within a training session and a marked decrement in performance on the first trial of the next day. It should be noted that with continued training, aged rats eventually do reach asymptote. However, it is unknown whether young rats with unilateral entorhinal cortex lesions would perform in a similar manner under the same training parameters.

A previous study by Foster et al. (1991) employed the same task parameters as those in the present study to examine cognitive decline during aging. When spatial discrimination training was massed into a single day, aged Fischer-344 rats acquired the spatial discrimination task to the same extent as young adults. Immediately following training, a probe trial indicated that both age groups exhibited similar spatially selective search behavior focused on the goal quadrant. However, when the same probe trial was administered $24 \mathrm{~h}$ later, only younger animals continued to search the goal quadrant. Together, the results suggest a similar cognitive deficit for aged rats and the UNI group in the present study, due to impairment in retention rather than in acquisition of a spatial search strategy.

Previous research has provided evidence for a distinction between involvement of the entorhinal cortex in the acquisition and retention of spatial information (Hunt, Kesner, \& Evans, 1994; Johnson \& Kesner, 1994) and sensory discrimination tasks (Levisohn \& Isacson, 1991; Myhrer \& Iversen, 1990; Staubli, Fraser, Kessler, \& Lynch, 1986; Vnek, Gleason, Kromer, \& Rothblat, 1995). In the case of sensory discrimination, lesioned animals tend to exhibit normal learning and impaired retention. Together, the results are consistent with the idea that entorhinal cortex damage influences retention performance for recently acquired information.

The extent of cortical damage or the degree of hippocampal afferent removal may interact with task demands to determine the range of cognitive impairments. Deficits in spatial discrimination on the water task following unilateral entorhinal cortex lesions have been reported for long (1-h) ITIs (Glasier, Sutton, \& Stein, 1995). However, in the Glasier et al. study, examination was conducted during the 7-16 days following surgery, an amount of time insufficient for completion of neuronal rearrangement thought to underlie behavioral recovery. In the present study, latency measures for acquisition of spatial discrimination indicated no difference between UNI and BIL groups, and only one trial block was different for UNI and CON rats. Although the data from the probe trialsa more valid measure of spatial learning -indicated that UNI animals learned the spatial task, the overall pattern of performance suggested that a slight impairment in the learning rate for the UNI group compared with the CON group. The most parsimonious explanation is that the remaining tissue or afferents were able to compensate to a certain extent in order to permit acquisition of the spatial task; however, the surviving fibers were unable to maintain information or permit retrieval over extended pe- riods, resulting in poor retention. The results emphasize the importance of examining retention as a cognitive constituent of the hippocampal-entorhinal system.

In aged rats, loss of perforant path input results in a decrease of the fiber potential and is associated with a reduction of the EPSP for a given stimulus intensity (Barnes \& McNaughton, 1980; Foster et al., 1991). However, the strength of surviving contacts, measured as the ratio of the EPSP and fiber potential responses, is enhanced for the medial perforant path of aged rats. The increased synaptic strength is due in part to an increase in postsynaptic responsiveness to the transmitter, suggesting the involvement of compensatory mechanisms for maintaining synaptic transmission (Barnes et al., 1992; Foster et al., 1991). Similarly, unilateral and bilateral entorhinal cortex lesions resulted in a decrease in perforant path fiber potentials and synaptic responses. Importantly, the extent of the reduction in the synaptic response was diminished for rats with unilateral relative to bilateral entorhinal cortex lesions. Furthermore, there was some indication that, as with aged rats, synaptic strength was increased for animals that received unilateral entorhinal cortex lesions.

In order to justify unilateral lesions as a model of aging, it will be important to determine whether the physiological mechanisms that underlie the growth of synaptic strength are similar across the two groups. It is becoming apparent that there are several mechanisms for regulating synaptic strength and that the capacity can be dynamically regulated by the number of synaptic contacts (G. Liu \& Tsien, 1995; Stewart, Schuster, Goodman, \& Atwood, 1996). Differences in the growth of synaptic strength could result from divergence in the ability to utilize various compensatory mechanisms. For example, the rate of collateral sprouting in response to removal of the perforant path is diminished during aging, possibly limiting sprouting as a form of compensation for afferent loss (Cotman \& Scheff, 1979). The negligible change in the EPSP/fiber potential ratio following bilateral lesions may be attributable to the extent of medial perforant path loss. The complete or nearly complete removal of the medial perforant path would permit proliferation of fiber collaterals in systems that do not activate excitatory ionotropic receptors (e.g., muscarinic cholinergic input from the medial septum) or that have a lower release probability, such as the lateral perforant path fibers (Christie \& Abraham, 1994; Colino \& Malenka, 1993; McNaughton, 1980). In the case of the UNI group, the attenuation of the lesion effect is consistent with previous work demonstrating growth of the synaptic response in vivo associated with sprouting of medial perforant path fibers from the contralateral entorhinal cortex (Steward et al., 1976). However, it is currently unknown whether the increase in synaptic strength for the UNI group was exclusively due to proliferation of synapses per afferent or, as in aged rats, involved an increase in the strength of individual synapses.

The results of the present study indicate that, as in aged rats, the growth of synaptic strength in lesioned rats 
is not due to an increase in transmitter release (Foster et al., 1991). The ratio of paired-pulse responses has been used as a measure of transmitter release, and the increase in this ratio for UNI animals is suggestive of a reduction in the probability of transmitter release. A reduction in the probability of transmitter release indicates a possible divergence in the physiological mechanisms between aged and UNI rats. A lower probability of release may be attributable to immaturity of newly formed synapses (Dumas \& Foster, 1995). However, synapse immaturity seems unlikely given the time between lesions and testing. Alternatively, the decrease in the paired-pulse ratio could result from proliferation of low-release lateral perforant path fibers from the intact ipsilateral perirhinal cortex into the dendritic zone abandoned by the medial perforant path (P. Liu \& Bilkey, 1996). Expansion of the ipsilateral lateral perforant path may be enhanced following the rapid and massive loss of the medial perforant path following a unilateral lesion. In contrast, scattered loss of the medial perforant path over the course of aging may restrict sprouting by the lateral perforant path. The present results suggest that the tendency for increased synaptic strength following unilateral entorhinal cortex removal is offset to a certain extent by a reduction in presynaptic function for some afferents, and the mechanism for reduction in transmitter release may indicate an important difference between the aging and the lesion models. Thus, future studies should consider more diffuse lesions or lesions that include the lateral perforant path input.

Partial removal of the entorhinal cortex appears to provide a better behavioral model of age-related spatial memory impairments and physiological adaptation to synapse loss than do more extensive lesions. This partial lesion model may provide a means for examining intervention treatments and may have important implications for agerelated changes observed in humans. Aging in humans and age-related diseases, such as Alzheimer's disease, are associated with damage to hippocampal afferents, including the entorhinal cortex (Heinsen et al., 1994; Hyman, Van Hoesen, Damasio, \& Barnes, 1984; Hyman, Van Hoesen, Kromer, \& Damasio, 1986), and characterized by an increased rate of forgetting for visuospatial information (Huppert \& Kopelman, 1989). However, it is still unclear what mechanism is responsible for the apparent retention deficit. Electrolytic lesions remove much more than just hippocampal afferents, and aging is associated with changes in hippocampal synaptic strength and plasticity in addition to afferent loss (Barnes, 1994; Geinisman et al., 1995; Norris, Korol, \& Foster, 1996). If the hippocampus acts to store information over the distribution of synaptic contacts, then removal of contacts would limit the storage capacity and may result in interference of stored information as newer information is presented over time. Alternatively, it may be that physiological changes, which are a direct result of synapse loss or are a result of decreased hippocampal transmission, result in consolidation or retrieval deficits. For example, the hippocampus appears to be involved in regulating stress responses (Sapolsky, Krey, \& McEwen, 1984), and there is considerable evidence that the level of stress-related hormones can regulate learning (Bodnoff et al., 1995; Bohus, Grubits, Kovacs, \& Lissak, 1970; de Wied \& Croiset, 1991; Gold \& Stone, 1988; Issa, Rowe, Gauthier, \& Meaney, 1990; McGaugh, 1989). Furthermore, aged humans and rodents exhibit an increased stress response that can generally impair learning performance (Fleg, Tzankoff, \& Lakatta, 1985; Hess \& Riegle, 1970; Lorens et al., 1990; Mabry et al., 1996; McCarty, 1986; Palmer, Ziegler, \& Lake, 1978; Sapolsky et al., 1984; Young, Rowe, Pallotta, Sparrow, \& Landsberg, 1980; Ziegler, Lake, \& Kopin, 1976). Thus, the possibility arises that loss of perforant path input may reflect alterations not only in the substrate for memory storage but also in processes that regulate memory function.

\section{REFERENCES}

BARNES, C. A. (1994). Normal aging: Regionally specific changes in hippocampal synaptic transmission. Trends in Neurosciences, 17, 13-18.

Barnes, C. A., \& McNaughton, B. L. (1980). Physiological compensation for loss of afferent synapses in rat hippocampal granule cells during senescence. Journal of Physiology, 309, 473-485.

Barnes, C. A., Rao, G., Foster, T. C., \& MCNaughton, B. L. (1992). Region-specific age effects on AMPA sensitivity: Electrophysiological evidence for loss of synaptic contacts in hippocampal field CA1. Hippocampus, 2, 457-468.

Bertoni-Freddari, C., Fattoretti, P., Pieroni, M., \& Meier-Ruge, W. (1992). Enlargement of synaptic size as a compensative reaction in aging and dementia. Pathology, Research \& Practice, 188, 612-615.

Bodnoff, S. R., Humphreys, A. G., Lehman, J. C., Dimond, D. M., Rose, G. M., \& MEANEY, M. J. (1995). Enduring effects of chronic corticosterone treatment on spatial learning, synaptic plasticity, and hippocampal neuropathology in young and mid-aged rats. Journal of Neuroscience, 15, 61-69.

Bohus, B., Grubits, J., Kovacs, G., \& Lissak, K. (1970). Effects of corticosteroids on passive avoidance behavior of rats. Acta Physiologica Academiae Scientiarum Hungaricae, 38, 381-391.

Christie, B. R., \& Abraham, C. (1994). Differential regulation of paired-pulse plasticity following LTP in the dentate gyrus. Neuroreport, 5, 385-388.

Colino, A., \& MalenKa, R. C. (1993). Mechanisms underlying induction of long-term potentiation in rat medial and lateral perforant paths in vitro. Journal of Neurophysiology, 69, 1150-1159.

Corman, C. W., \& Scheff, S. W. (1979). Compensatory synaptic growth in aged animals after neuronal death. Mechanisms of Aging \& Development, 9, 103-117.

DE WIED, D., \& CROISET, G. (1991). Stress modulation of learning and memory processes. Methods \& Achievements in Experimental Pathology, 15, 167-199.

Diana, G., Domenici, M. R., Scotti de Carolis, A., Loizzo, A., \& SAGRATELla, S. (1995). Reduced hippocampal CA1 Ca2t-induced long-term potentiation is associated with age-dependent impairment of spatial learning. Brain Research, 686, 107-110.

Dumas, T. C., \& Foster, T. C. (1995). Developmental increase in CA3$\mathrm{CAl}$ presynaptic function in the hippocampal slice. Journal of Neurophysiology, 73, 1821-1828.

Fleg, J. L., Tzankoff, S. P., \& Lakatta, E. G. (1985). Age-related augmentation of plasma catecholamines during dynamic exercise in healthy males. Journal of Applied Physiology, 59, 1033-1039.

Foster, T. C., Barnes, C. A., RaO, G., \& MCNaughton, B. L. (1991). Increase in perforant path quantal size in aged F-344 rats. Neurobiology of Aging, 12, 441-448.

Foster, T. C., Gagne, J., \& MassicotTe, G. (1996). Mechanism of altered synaptic strength due to experience: Relation to long-term potentiation. Brain Research, 736, 243-250. 
Gage, F. H., DunNetT, S. B., \& BJorklund, A. (1984). Spatial learning and motor deficits in aged rats. Neurobiology of Aging, 5, 43-48.

Geddes, J. W., Monaghan, D. T., Cotman, C. W., LotT, I. T., Kim, R. C., \& ChUi, H. C. (1985). Plasticity of hippocampal circuitry in Alzheimer's disease. Science, 230, 1179-1181.

Geinisman, Y., deToledo-Morrell, L., Morrell, F., \& Heller, R. E. (1995). Hippocampal markers of age-related memory dysfunction: Behavioral, electrophysiological and morphological perspectives. Progress in Neurobiology, 45, 223-252.

Glasier, M. M., Sutton, R. L., \& Stein, D. G. (1995). Effects of unilateral entorhinal cortex lesion and ganglioside GM1 treatment on performance in a novel water maze task. Neurobiology of Learning \& Memory, 64, 203-214.

Gold, P. E., McGaugh, J. L., Hankins, L. L., Rose, R. P., \& Vasquez, B. J. (1981). Age dependent changes in retention in rats. Experimental Aging Research, 8, 53-58.

Gold, P. E., \& STONE, W. S. (1988). Neuroendocrine factors in agerelated memory dysfunctions: Studies in animals and humans. Neurobiology of Aging, 9, 709-717.

Hagan, J. J., VerheiJck, E. E., Spigt, M. H., \& Ruigt, G. S. (1992). Behavioural and electrophysiological studies of entorhinal cortex lesions in the rat. Physiology \& Behavior, 51, 255-266.

Heinsen, H., Henn, R., Eisenmenger, W., Gotz, M., Bohl, J., Bethke, B., LockemanN, U., \& Puschel, K. (1994). Quantitative investigations on the human entorhinal area: Left-right asymmetry and age-related changes. Anatomy \& Embryology, 190, 181-194.

Hess, G., \& Riegle, G. (1970). Chronic and acute dexamethasone suppression of stress activation of the adrenal cortex in young and aged rats. Neuroendocrinology, 9, 175-187.

Hunt, M. E., Kesner, R. P., \& Evans, R. B. (1994). Memory for spatial location: Functional dissociation of entorhinal cortex and hippocampus. Psychobiology, 22, 186-194.

HUPPERT, F. A., \& Kopelman, M. D. (1989). Rates of forgetting in normal aging: A comparison with dementia. Neuropsychologia, 27, 849-869.

hyman, B. T., Kromer, L. J., \& Van Hoesen, G. W. (1987). Reinnervation of the hippocampal perforant pathway zone in Alzheimer's disease. Annals of Neurology, 21, 259-267.

Hyman, B. T., Van Hoesen, G. W., Damasio, A. R., \& Barnes, C. L. (1984). Alzheimer's disease: Cell-specific pathology isolates the hippocampal formation. Science, 225, 1168-1170.

hyman, B. T., Van Hoesen, G. W., Kromer, L. J., \& Damasio, A. R. (1986). Perforant pathway changes and the memory impairment of Alzheimer's disease. Annals of Neurology, 20, 472-481.

Issa, A. M., Rowe, W., Gauthier, S., \& Meaney, M. J. (1990). Hypothalamic-pituitary-adrenal activity in aged, cognitively impaired and cognitively unimpaired rats. Journal of Neuroscience, 10, $3247-3254$.

Johnson, D. L., \& KeSner, R. P. (1994). The effects of lesions of the entorhinal cortex and the horizontal nucleus of the diagonal band of Broca upon performance of a spatial location recognition task. Behavioural Brain Research, 61, 1-8.

LEVISOHN, L. F., \& ISACSON, O. (1991). Excitotoxic lesions of the rat entorhinal cortex effects of selective neuronal damage on acquisition and retention of a nonspatial reference memory task. Brain Research, $564,230-244$

Linder, M. D., Balch, A. H., \& VanderMaelen, C. P. (1992). Short forms of the "reference-" and "working-memory" Morris water maze for assessing age-related deficits. Behavioral \& Neural Biology, 58, 94-102.

Linder, M. D., \& GRIBKoFF, V. K. (1991). The relationship of visual acuity and thermoregulatory function to age-related deficits in the Morris water task. Behavioural Brain Research, 45, 45-55.

Liv, G., \& Tsien, R. W. (1995). Properties of synaptic transmission at single hippocampal synaptic buttons. Nature, 375, 404-408.

LiU, P., \& BILKEY, D. K. (1996). Direct connection between perirhinal cortex and hippocampus is a major constituent of the lateral perforant path. Hippocampus, 6, 125-135.

LOESCHE, J., \& STEWARD, O. (1977). Behavioral correlates of denervation and reinnervation of the hippocampal formation of the rat: Re- covery of alternation performance following unilateral entorhinal cortex lesions. Brain Research Bulletin, 2, 31-39.

Lorens, S. A., Hata, N., Van De Kar, L. D., Guschwan, M., Goral, J., Lee, J. M., Hamilton, M. E., Bethea, C., \& Clancy, J., Jr. (1990). Neurochemical, endocrine and immunological responses to stress in young and old Fischer 344 male rats. Neurobiology of Aging, 11, 139-150.

Lynch, G., Gall, C., Rose, G., \& Cotman, C. (1976). Changes in the distribution of the dentate gyrus associational system following unilateral or bilateral entorhinal lesions in adult rat. Brain Research, 110, $57-71$.

Mabry, T. R., McCarty, R., Gold, P. E., \& Foster, T. C. (1996). Age and stress-history effects on spatial performance in a swim task in Fischer-344 rats. Neurobiology of Learning \& Memory, 66, 1-10.

MARTINEZ, J. L., \& RigTer, H. (1980). Endorphins alter acquisition and consolidation of an inhibitory avoidance response in rats. Neuroscience Letters, 19, 197-201.

MCCARTY, R. (1986). Age-related alterations in sympathetic-adrenal medullary responses to stress. Gerontology, 32, 172-183.

MCGAUGH, J. L. (1989). Involvement of hormonal and neuromodulatory systems in the regulation of memory storage. Annual Review of Neuroscience, 12, 255-287.

MCNaughton, B. L. (1980). Evidence for two physiologically distinct perforant pathways to the fascia dentata. Brain Research, 199, 1-19.

Moser, E. I. (1996). Altered inhibition of dentate granule cells during spatial learning in an exploration task. Journal of Neuroscience, 16, 1247-1259.

MyHrer, T., \& IVERSEN, E. G. (1990). Changes in retention of a visualdiscrimination task following unilateral and bilateral transections of temporoentorhinal connections in rats. Brain Research Bulletin, 25, 293-298.

Nagahara, A. H., OtTo, T., \& Gallagher, M. (1995). Entorhinalperirhinal lesions impair performance of rats on two versions of place learning in the Morris water task. Behavioral Neuroscience, 109, 3-9.

Norris, C. M., KoroL, D. L., \& Foster, T. C. (1996). Increased susceptibility to induction of long-term depression and long-term potentiation reversal during aging. Journal of Neuroscience, 16, 53825392 .

Olton, D. S., \& Wenk, G. L. (1987). Dementia: Animal models of the cognitive impairments produced by degeneration of the basal forebrain cholinergic system. In H. Y. Meltzer (Ed.), Psychopharmacology: The third generation of progress (pp. 941-953). New York: Raven Press.

Palmer, G. J., Ziegler, M. G., \& LaKe, C. R. (1978). Response of norepinephrine and blood pressure to stress increases with age. Journal of Gerontology, 33, 482-487.

RapP, P. R., Rosenberg, R. A., \& Gallagher, M. (1987). An evaluation of spatial information processing in aged rats. Behavioral Neuroscience, 101, 3-12.

Rasmussen, T., Schliemann, T., Sørensen, J. C., Zimmer, J., \& West, M. J. (1996). Memory-impaired aged rats-No loss of principal hippocampal and subicular neurons. Neurobiology of Aging, 17, 143-147.

ReEves, T. M., \& SMITH, D. C. (1987). Reinnervation of the dentate gyrus and recovery of alternation behavior following entorhinal cortex lesions. Behavioral Neuroscience, 101, 179-186.

SAPOLSKY, R. M., KREY, L. C., \& MCEWEN, B. S. (1984). Glucocorticoid-sensitive hippocampal neurons are involved in terminating the adrenocortical stress response. Proceedings of the National Academy of Sciences, 81, 6174-6177.

SchefF, S. W., Bernardo, L. S., \& Cotman, C. W. (1980). Decline in reactive fiber growth in the dentate gyrus of aged rats compared to young adult rats following entorhinal cortex removal. Brain Research, 199, $21-38$

SCHENK, F., \& MORRIS, R. G. M. (1985). Dissociation of components of spatial memory in rats after recovery from the effects of retrohippocampal lesions. Experimental Brain Research, 58, 11-28.

Skelton, R. W., \& MCNAMARA, R. K. (1992). Bilateral knife cuts to the perforant path disrupt spatial learning in the Morris water maze. Hippocampus, 2, 73-80.

Solomon, P. R., \& Pendlebury, W. W. (1994). A model systems ap- 
proach to the study of disorders of learning and memory. Neurobiology of Aging, 15, 283-286.

Staubli, U., Fraser, D., Kessler, M., \& Lynch, G. (1986). Studies in retrograde and anterograde amnesia of olfactory memory after denervation of the hippocampus by entorhinal cortex lesions. Behavioral \& Neural Biology, 46, 432-444.

Steward, O., Cotman, C. W., \& LYNCH, G. (1976). A quantitative audioradiographic analysis of the time course of proliferation of contralateral entorhinal cortex following ipsilateral entorhinal lesions. Brain Research, 114, 181-200.

Stewart, B. A., Schuster, C. M., Goodman, C. S., \& Atwood, H. L. (1996). Homeostasis of synaptic transmission in Drosophila with genetically altered nerve terminal morphology. Journal of Neuroscience, 16, 3877-3886.

VNek, N., Gleason, T., Kromer, L. F., \& Rothblat, L. A. (1995).
Entorhinal-hippocampal connections and object memory in the rat: Acquisition versus retention. Journal of Neuroscience, 15, 3193-3199.

WinocUR, G. (1988). A neuropsychological analysis of memory loss with age. Neurobiology of Aging, 9, 487-494.

Young, J. B., Rowe, J. W., Pallotta, J. A., Sparrow, D., \& LandsBERG, L. (1980). Enhanced plasma norepinephrine response to upright posture and oral glucose administration in elderly human subjects. Metabolism, 29, 532-539.

Ziegler, M. G., LAKE, C. R., \& KoPIN, I. J. (1976). Plasma noradrenaline increases with age. Nature, 261, 333-334.

ZORNETZER, S. F., ThOMPSON, R., \& ROgerS, J. (1982). Rapid forgetting in aged rats. Behavioral \& Neural Biology, 36, 49-60.

(Manuscript received March 11, 1997; revision accepted for publication May 22, 1997.) 